Internist 2015 $\cdot 56: 80-83$

DOI 10.1007/s00108-014-3624-5

Online publiziert: 14. Januar 2015

(c) Springer-Verlag Berlin Heidelberg 2015

\section{Rubrikherausgeber:}

K. Werdan, Halle (Saale)

\author{
S. Pickel ${ }^{1} \cdot$ M. Filipowicz ${ }^{2}$ E. Bruder ${ }^{3} \cdot$ M. Battegay ${ }^{4,5} \cdot$ M. Osthoff $^{4,5}$ \\ ${ }^{1}$ Klinik für Innere Medizin, Universitätsspital Basel \\ ${ }^{2}$ Klinik für Gastroenterologie und Hepatologie, Universitätsspital Basel \\ ${ }^{3}$ Pathologie, Universitätsspital Basel \\ ${ }^{4}$ Klinik für Infektiologie und Spitalhygiene, Universitätsspital Basel \\ ${ }^{5}$ Departement Klinische Forschung, Universitätsspital Basel
}

\title{
Gewichtsverlust und chronische Diarrhö bei einem 54-jährigen Mann mit HIV-Infektion
}

\section{Anamnese}

Ein in der Schweiz aufgewachsener 54-jähriger Patient stellte sich im April 2014 erstmalig ambulant in unserer Sprechstunde vor. Er berichtete über flüssigen, nicht blutigen Stuhlgang mit einer Frequenz von bis zu 5-mal pro Tag seit seiner Rückkehr aus einem Thailandurlaub im Februar 2014. Außerdem klagte der Patient über Appetitlosigkeit mit Nausea und einen Gewichtsverlust von $11 \mathrm{~kg}$ in 6 Wochen. Nachts traten subfebrile Temperaturen auf. Weitere Beschwerden wie Erbrechen, Nachtschweiß, Husten, Dyspnoe, Schluckbeschwerden oder Dysurie wurden verneint.

Der Hausarzt hatte Ende März 2014 bereits eine Shigellose diagnostiziert und diese mit Ciprofloxacin $500 \mathrm{mg}$ 2-mal täglich p.o. über 5 Tage resistenzgerecht behandelt. Eine relevante Befundbesserung blieb jedoch aus.

In der Vorgeschichte war eine HIVInfektion seit etwa 2005 bekannt. Diesbezüglich war der homosexuelle Patient bisher nicht in ärztlicher Betreuung. Umstände und Zeitpunkt der Infektion waren unklar. Er gab ungeschützten Geschlechtsverkehr mit wechselnden Partnern an, zuletzt in Thailand.

Weitere relevante Vorerkrankungen waren eine lege artis therapierte Syphilis im Stadium II (2008), ein monosegmentaler thorakaler Herpes zoster (2012), eine Hepatitis A (Kindesalter) und B (1999) sowie eine Gonorrhö (etwa 2003).

Noxen, Allergien und die regelmäßige Einnahme von Medikamenten wur- den verneint. Der Patient ist kaufmännischer Angestellter in einem großen Unternehmen.

\section{Befunde}

\section{Klinischer Untersuchungsbefund}

Der 54-jährige Patient war bei Erstvorstellung in einem reduzierten Allgemeinzustand. Der Body-Mass-Index betrug $20,0 \mathrm{~kg} / \mathrm{m}^{2}$ bei einer Körpergröße von $183 \mathrm{~cm}$ und einem Körpergewicht von $67 \mathrm{~kg}$. Der Blutdruck lag bei 114/69 mmHg, die Herzfrequenz bei 76/ min, die Temperatur bei $38,1^{\circ} \mathrm{C}$ und die Atemfrequenz bei $16 / \mathrm{min}$. Haut und Schleimhäute waren unauffällig. Die Halsvenen waren kollabiert. Vergrößerte Lymphknoten waren nicht palpabel. Die Untersuchung von Herz, Lunge und Abdomen war unauffällig. In der digitalrektalen Tastuntersuchung fand sich gelblicher, weicher Stuhlgang.

\section{Laborchemische Untersuchungen}

\section{Blutbild und klinische Chemie}

Laborchemisch zeigten sich eine normochrome, normozytäre Anämie, eine Hyponatriämie und Hypokaliämie sowie eine leichte Erhöhung des C-reaktiven Proteins und der alkalischen Phosphatase (• Tab. 1). Die CD4-Zellen waren mit 106 Zellen/ $\mu \mathrm{l}(9 \%)$ deutlich erniedrigt (Normbereich: 700-1100 Zellen/ $\mu \mathrm{l}$ ).

\section{Serologie und Virologie}

Die HI-Viruslast lag bei 59.345 Kopien/ $\mathrm{ml}$. Folgende Serologien waren mit einer zurückliegenden Infektion vereinbar:

- Zytomegalievirus (CMV)

- Toxoplasmose

- Syphilis

- Hepatitis A und B.

Die Hepatitis-C-Serologie war negativ. Der Interferon- $\gamma$-release-Assay (T-SPOT. TB-Test ${ }^{\bullet}$ ) war positiv.
Tab. 1 Laborwerte bei Erstvorstellung und eine Woche später

\begin{tabular}{llll}
\hline Laborwert (Einheit) & Normbereich & Erstvorstellung & 1 Woche später \\
\hline Hämoglobin $(\mathrm{g} / \mathrm{l})$ & $140-180$ & 101 & 104 \\
\hline Leukozyten $\left(10^{9} / \mathrm{l}\right)$ & $3,50-10,00$ & 6,77 & 6,09 \\
\hline Thrombozyten $\left(10^{9} / \mathrm{l}\right)$ & $150-450$ & 435 & 531 \\
\hline C-reaktives Protein $(\mathrm{mg} / \mathrm{l})$ & $<10$ & 63 & 154 \\
\hline Kreatinin $(\mu \mathrm{mol} / \mathrm{l})$ & $49-97$ & 68 & 90 \\
\hline Serumnatrium $(\mathrm{mmol} / \mathrm{l})$ & $135-145$ & 126 & 120 \\
\hline Serumkalium $(\mathrm{mmol} / \mathrm{l})$ & $3,8-4,8$ & 3,0 & 3,0 \\
\hline Alkalische Phosphatase $(\mathrm{U} / \mathrm{l})$ & $<129$ & 178 & 253
\end{tabular}



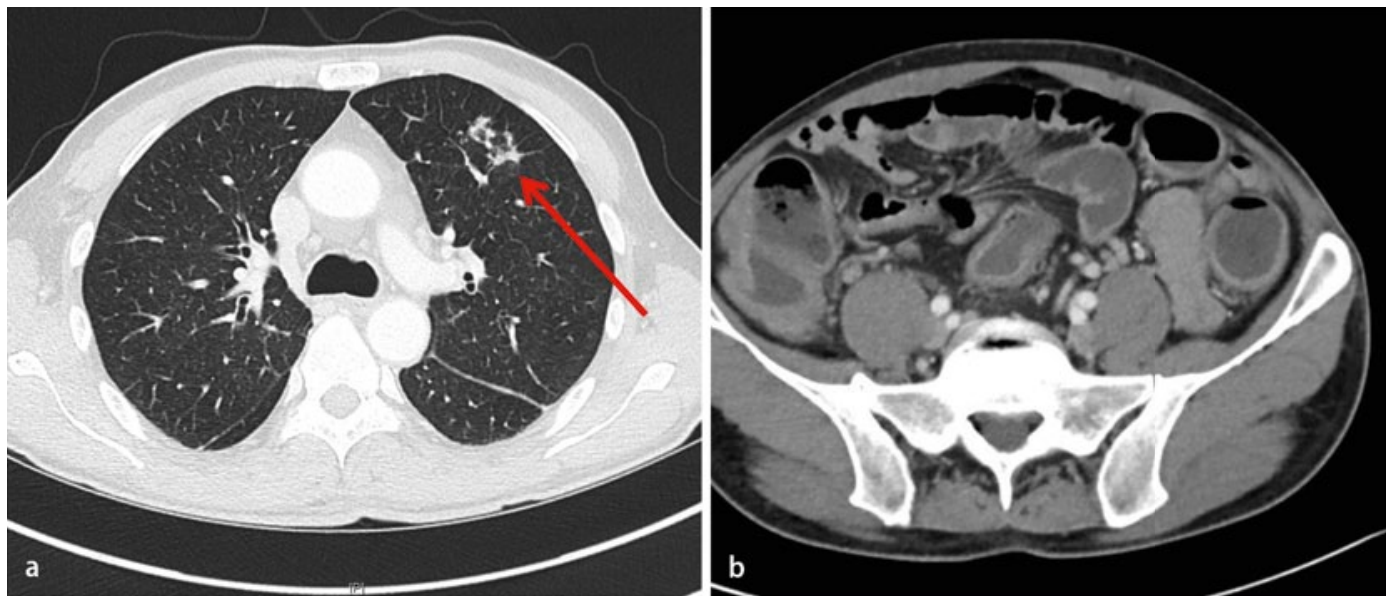

Abb. $1<$ Computertomographie. a Atypische Infiltrate im linken Oberlappensegment (Pfeile). b Wandverdickung des gesamten Kolonrahmens

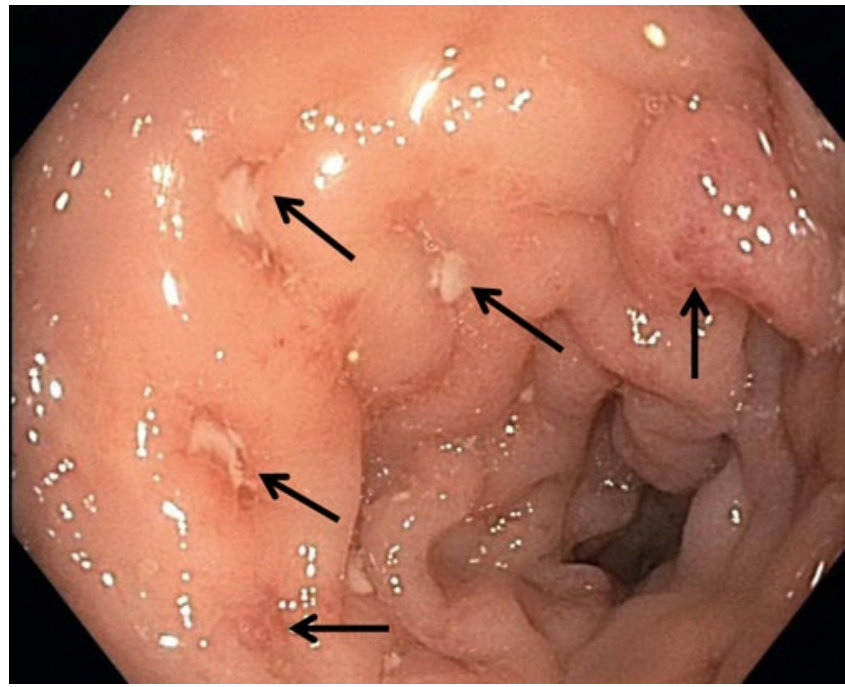

Abb. $2<$ Koloskopie. Gerötete Mukosa, Erosionen und Ulzerationen (Pfeile)

\section{Verdachtsdiagnosen}

\section{Infektiös:}

- Wasting-Syndrom bei unbehandelter HIV-Infektion

- Infektiöse Enteritis oder Kolitis (Differenzialdiagnosen: insbesondere CMV, Salmonellen, Clostridium difficile, Entamoeba histolytica, atypische Mykobakterien, Kryptosporidien)

- Tuberkulose

Nichtinfektiös:

- Lymphom

- Medikamentös (z. B. Proteaseinhibitoren, Antibiotika)

\section{Weitere Diagnostik und primärer Verlauf}

In der Stuhldiagnostik konnte mikroskopisch Strongyloides stercoralis nachgewie- sen werden. Die gleichzeitig abgenommenen serologischen Proben fielen ebenso positiv aus. Eine Therapie mit Ivermectin $0,2 \mathrm{mg} / \mathrm{kgKG}$ an den Tagen 1 und 14 wurde durchgeführt. Eine Stuhluntersuchung im Verlauf fiel negativ aus.

Bezüglich der weit fortgeschrittenen HIV-Infektion willigte der Patient in die Einleitung einer antiretroviralen Therapie (ART) mit Tenofovir, Emtricitabin und Ritonavir-geboostetem Darunavir ein. Bei einer CD4-Zellzahl unter 200/ $\mu$ l wurde außerdem eine primärprophylaktische Therapie gegen Pneumocystis jirovecii und Toxoplasma gondii mit Cotrimoxazol 800/160 mg 3-mal pro Woche begonnen. Bei progredienter Verschlechterung des Allgemeinzustands mit persistierender Diarrhö und subfebrilen Temperaturen erfolgte eine Woche später die stationäre Aufnahme (Laborbefunde s. - Tab. 1). Unter i.v.-Rehydrierung bes- serten sich der Allgemeinzustand und die Elektrolytentgleisung schnell.

Computertomographisch zeigten sich atypische Infiltrate im linken Oberlappensegment und Zeichen einer Kolitis mit mesenterialer Lymphadenopathie (• Abb. 1).

Zur weiteren Diagnostik bei Verdacht auf einen pulmonalen opportunistischen Infekt wurde eine Bronchoskopie mit bronchoalveolärer Lavage (BAL) durchgeführt. Untersuchungen auf aerobe oder anaerobe Bakterien, respiratorische Viren, Pneumocystis jirovecii und Schimmelpilze fielen negativ aus. Mikroskopisch konnten weder in der BAL noch in drei Sputa säurefeste Stäbchen nachgewiesen werden. Ebenso war die Polymerase-Kettenreaktion (PCR) auf den Mycobacterium-tuberculosis-Komplex negativ. Bei weiterhin persistierender Diarrhö führten wir eine Koloskopie durch, in der sich makroskopisch das Bild einer ulzerierenden Kolitis zeigte (• Abb. 2). Histologisch fand sich in den Darmbiopsien eine hochgradig aktive Kolitis mit Nachweis von CMV in der Immunhistochemie (• Abb. 3) und PCR (826.194 Kopien/ml). Die CMV-PCR im Serum war negativ. Eine CMV-Retinitis konnte nicht nachgewiesen werden.

\section{Diagnosen}

- CMV-Kolitis bei HIV-Infektion (Stadium CDC C3)

- Strongyloidose und Shigellenenteritis nach Thailandaufenthalt

- Unklare pulmonale Infiltrate (Differenzialdiagnosen: Mykobakterien, Nocardien, Pilze) 


\section{Therapie und Verlauf 1}

Der Patient war bereits vor Vorliegen der Diagnose einer CMV-Kolitis bei sistierter Diarrhö entlassen worden. Nach Erhalt der histologischen Resultate wurde die CMV-Kolitis bei fast vollständiger Beschwerdefreiheit mit Valganciclovir 900 mg 2-mal täglich p.o. über 2 Wochen therapiert. Daran schloss sich eine Erhaltungstherapie mit 900 mg 1-mal täglich an.

Bezüglich der HIV-Infektion zeigte sich nach 4-wöchiger Therapie bereits ein Anstieg der CD4-Zellen auf 181/ $\mu$ l (11\%) und ein Abfall der HIV-Last auf 347 Kopien $/ \mathrm{ml}$.

Im weiteren Verlauf war der Patient beschwerdefrei. Er nahm rasch an Gewicht zu und wiegt aktuell $75 \mathrm{~kg}$. Zwei Wochen nach Entlassung des Patienten wurde allerdings ein Wachstum von Mykobakterien in der bronchoalveolären Probe festgestellt. Mittels PCR konnte Mycobacterium tuberculosis identifiziert werden. Zusätzlich ergab sich in der Schnell-PCR (GeneXpert MTB/RIF ${ }^{\otimes}$ ) der Verdacht auf eine Rifampicin-Mutation.

\section{Definitive Diagnosen}

\section{- CMV-Kolitis bei HIV-Infektion (Sta- dium CDC C3) \\ - Strongyloidose und Shigellenenteritis nach Thailandaufenthalt - Multiresistente Lungentuberkulose}

\section{Therapie und Verlauf 2}

Seit Juni 2014 wird der Patient bei bestätigter multiresistenter Tuberkulose mit einer tuberkulostatischen Fünffachtherapie behandelt. Nach 2 Monaten waren die Infiltrate in der Computertomographie diskret regredient. Geplant ist eine Therapiedauer von mindestens 18 Monaten.

\section{Diskussion}

\section{Unbehandelte HIV-Infektion}

Bei dem vorgestellten Patienten lag seit 2005 eine unbehandelte HIV-Infektion vor. In Deutschland und der Schweiz ist der Anteil der Patienten, deren Erkrankung in einem fortgeschrittenen Stadium diagnostiziert wird (CD4-Zellen <350/ $\mu \mathrm{l}$ bzw. AIDS-definierende Erkrankung), mit 49,5\% [1] bzw. 45\% [2] vergleichbar hoch. Die Zahl derer, die sich spät in medizinische Betreuung begeben, ist in Deutschland mit 58,1\% sogar noch höher [1]. Als Risikofaktoren für eine späte HIV-Diagnosestellung gelten höheres Alter, Heterosexualität und ein Migrationshintergrund. Ein deutlicher Rückgang war in den letzten Jahren dagegen bei homosexuellen Männern zu verzeichnen $[1,3]$.

Eine späte Diagnosestellung und ein später Therapiebeginn sind mit einer höheren Mortalität assoziiert $[4,5]$. Eine frühzeitige Diagnosestellung und ein rechtzeitiger Therapiebeginn sind entscheidend. Patienten, deren Erkrankung in einem fortgeschrittenen Stadium diagnostiziert wird, stellen sich mit einer Vielzahl unspezifischer Symptome vor, denen aber oft eine relevante opportunistische Erkrankung zugrunde liegt. Gewichtsverlust und Diarrhö, wie bei dem von uns vorgestellten Patienten, treten bei 35\% bzw. 16\% der unbehandelten HIV-infizierten Patienten auf [2]. Differenzialdiagnostisch kommt eine Vielzahl infektiöser und nichtinfektiöser Erkrankungen in Betracht.

\section{CMV-assoziierte \\ Organerkrankungen bei \\ Patienten mit HIV}

Vor Einführung der ART waren CMV-assoziierte Organerkrankungen häufig. Zudem waren sie mit einer schlechten Prognose assoziiert [6]. Seit Einführung der ART ist auch in Deutschland ein deutlicher Rückgang der Inzidenz zu verzeichnen [7].

\section{\) Die Kolitis ist eine seltene Manifestation der CMV-Infektion bei HIV-Infizierten Patienten}

CMV-Infektionen können sich in verschiedenen Organsystemen manifestieren, mit Abstand am häufigsten ist die Retinitis. Die CMV-Kolitis ist eine seltene Manifestation, die sich klinisch mit blutiger oder unblutiger Diarrhö begleitet von Gewichtsverlust, Fieber und Bauchschmerzen darstellt. Zur Diagnosestel-
Internist 2015 $\cdot 56: 80-83$

DOI 10.1007/s00108-014-3624-5

(c) Springer-Verlag Berlin Heidelberg 2015

\section{S. Pickel · M. Filipowicz · E. Bruder · M. Battegay - M. Osthoff \\ Gewichtsverlust und chronische Diarrhö bei einem 54-jährigen Mann mit HIV-Infektion}

\section{Zusammenfassung}

Ein 54-jähriger Mann stellte sich mit seit 6 Wochen bestehender chronischer Diarrhö und einem Gewichtsverlust von $11 \mathrm{~kg}$ nach Thailandaufenthalt vor. Es lag eine dem Patienten seit 9 Jahren bekannte, bisher nicht behandelte und fortgeschrittene HIV-Erkrankung vor. Die Therapie einer im Verlauf diagnostizierten Shigellose und Strongyloidose führte zu keiner Symptombesserung. Nach Koloskopie wurde schließlich eine Zytomegalieviruskolitis diagnostiziert. Unter antiretroviraler Therapie und Therapie mit Valganciclovir kam es schnell zu einer Befundbesserung. Als weitere opportunistische Erkrankung wurde eine multiresistente pulmonale Tuberkulose nachgewiesen.

\section{Schlüsselwörter}

Kolitis - Zytomegalievirus - Opportunistische Infektion · Strongyloidose · Shigellose

\section{Weight loss and chronic diarrhea in a 54-year-old man with HIV infection}

\section{Abstract}

A 54-year-old man presented with a 6-week history of chronic diarrhea and weight loss of $11 \mathrm{~kg}$ after returning from a holiday in Thailand. The patient had a 9-year history of an untreated HIV infection. Despite treatment of a culture-proven Shigella enteritis and strongyloidiasis the symptoms persisted. Finally, cytomegalovirus (CMV) colitis was diagnosed by colonoscopy. The patient recovered completely after starting antiretroviral and valganciclovir treatment. An additional opportunistic infection with multiresistant pulmonary tuberculosis was diagnosed.

\section{Keywords}

Colitis · Cytomegalovirus · Opportunistic infection · Strongyloidiasis · Shigellosis

lung ist in der Regel eine Koloskopie mit Biopsie und immunhistochemischem Nachweis von CMV-Einschlusskörperchen notwendig, eine negative CMV-PCR schließt eine CMV-Kolitis nicht aus [8]. 


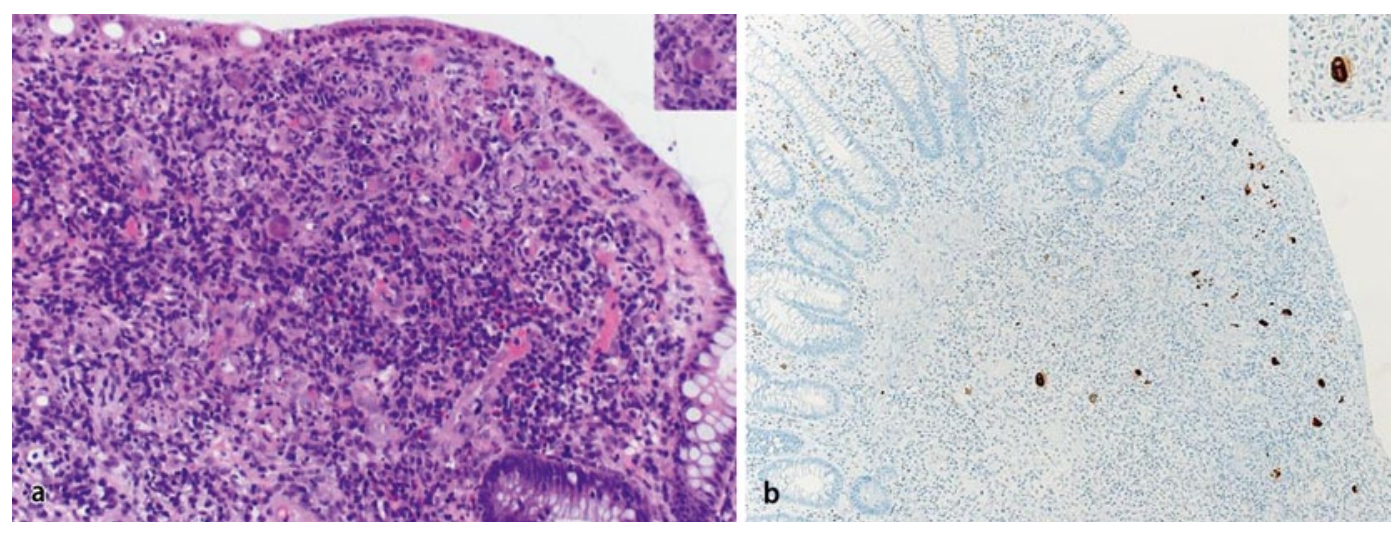

Abb. $3 \Delta$ Histologie. a Floride Kolitis mit Kryptenverlust und viruszytopathischen Veränderungen (ballonierte Zellen mit zytopathischem Effekt und stark eosinophilen zytoplasmatischen sowie nukleären Viruseinschlusskörpern (,inset“; HE-Färbung, Vergr. 200:1). b Immunhistochemische Färbung (Braunfärbung) gegen CMV beweist die Präsenz von CMV in den histologisch suspekten Zellen („,inset; CMV-Immunhistochemie, Vergr. 100:1). CMV Zytomegalievirus

Als Standardtherapie bei CMV-Kolitis wird prinzipiell eine i.v.-Therapie mit Ganciclovir 2-mal $5 \mathrm{mg} / \mathrm{kgKG} /$ Tag über 3 Wochen empfohlen, bei milder klinischer Ausprägung alternativ eine Behandlung mit Valganciclovir oral 2-mal $900 \mathrm{mg}$. Eine Sekundärprophylaxe wird nicht empfohlen. Voraussetzung für einen dauerhaften Therapieerfolg bei der CMVKolitis ist eine Immunrekonstitution durch eine erfolgreiche ART, die per se zu einer signifikanten Abnahme der CMVViruslast führt $[9,10]$. Der Patient war 4 Wochen nach Beginn einer ART und noch vor Beginn der eigentlichen CMVTherapie bereits fast beschwerdefrei.

Neben der CMV-Kolitis konnten wir bei dem vorgestellten Patienten eine Shigellose und eine Strongyloidose diagnostizieren. Während eine HIV-Erkrankung bzw. Homosexualität keine Risikofaktoren für eine Strongyloidose sind, tritt eine Shigellose gehäuft bei HIV-infizierten Patienten und bei homosexuellen Männern auf [11].

Als weitere opportunistische Erkrankung konnten wir bei unserem Patienten eine pulmonale Tuberkulose diagnostizieren. Dieser Fall zeigt exemplarisch, dass Patienten mit später Diagnosestellung bzw. spätem Therapiebeginn gleichzeitig an mehreren Erkrankungen leiden können.

\section{Fazit für die Praxis}

Die HIV-Erkrankung wird auch heute noch in knapp 50\% der Fälle erst in einem fortgeschrittenen Stadium diag- nostiziert. Die Patienten können sich mit mehreren opportunistischen und - wie in unserer Kasuistik- reiseassoziierten Erkrankungen vorstellen. Bei Symptompersistenz sollte folglich eine weitere gezielte Abklärung erfolgen.

Der frühe Beginn einer ART verbessert die Prognose signifikant und ist bei opportunistischen Infektionen Voraussetzung für einen anhaltenden Therapieerfolg.

\section{Korrespondenzadresse}

\section{Dr. M. Osthoff}

Klinik für Infektiologie und Spitalhygiene, Universitätsspital Basel

Petersgraben 4, 4031 Basel

Schweiz

michael.osthoff@usb.ch

\section{Einhaltung ethischer Richtlinien}

Interessenkonflikt. S. Pickel, M. Filipowicz, E. Bruder, M. Battegay und M. Osthoff geben an, dass kein Interessenkonflikt besteht.

Alle Patienten, die über Bildmaterial oder anderweitige Angaben innerhalb des Manuskripts zu identifizieren sind, haben hierzu ihre schriftliche Einwilligung gegeben. Im Falle von nicht mündigen Patienten liegt die Einwilligung eines Erziehungsberechtigen oder des gesetzlich bestellten Betreuers vor.

\section{Literatur}

1. Zoufaly A, an der Heiden M, Marcus U et al (2012) Late presentation for HIV diagnosis and care in Germany. HIV Med 13(3):172-181

2. Buetikofer S, Wandeler G, Kouyos R et al (2014) Prevalence and risk factors of late presentation for HIV diagnosis and care in a tertiary referral centre in Switzerland. Swiss Med Wkly 144:w13961
3. Wolbers M, Bucher HC, Furrer $\mathrm{H}$ et al (2008) Delayed diagnosis of HIV infection and late initiation of antiretroviral therapy in the Swiss HIV Cohort Study. HIV Med 9(6):397-405

4. Kitahata MM, Gange SJ, Abraham AG et al (2009) Effect of early versus deferred antiretroviral therapy for HIV on survival. N Engl J Med 360(18):18151826

5. May M, Gompels M, Delpech V et al (2011) Impact of late diagnosis and treatment on life expectancy in people with HIV-1: UK Collaborative HIV Cohort (UK CHIC) Study. BMJ 343:d6016

6. Gallant JE, Moore RD, Richman DD et al (1992) Incidence and natural history of cytomegalovirus disease in patients with advanced human immunodeficiency virus disease treated with zidovudine. The Zidovudine Epidemiology Study Group. J Infect Dis 166(6):1223-1227

7. Salzberger B, Hartmann P, Hanses F et al (2005) Incidence and prognosis of $\mathrm{CMV}$ disease in HIV-infected patients before and after introduction of combination antiretroviral therapy. Infection 33(56):345-349

8. Yan Z, Wang L, Dennis J et al (2014) Clinical significance of isolated cytomegalovirus-infected gastrointestinal cells. Int J Surg Pathol 22(6):492-498

9. O'Sullivan CE, Drew WL, McMullen DJ et al (1999) Decrease of cytomegalovirus replication in human immunodeficiency virus infected-patients after treatment with highly active antiretroviral therapy. $J$ Infect Dis 180(3):847-849

10. Deayton J, Mocroft A, Wilson P et al (1999) Loss of cytomegalovirus (CMV) viraemia following highly active antiretroviral therapy in the absence of specific anti-CMV therapy. Aids 13(10):1203-1206

11. Aragón TJ, Vugia DJ, Shallow S et al (2007) Casecontrol study of shigellosis in San Francisco: the role of sexual transmission and HIV infection. Clin Infect Dis 44(3):327-334 